\title{
Tecnologías y sistemas de información en la gestión de conocimiento en las organizaciones
}

\author{
Andrade Castro, Jesús Alberto*- \\ * Economista. Magister en Computación Aplicada y Master in Management Information \\ Systems. Profesor Titular del Departamento de Computación. Director del Departamento \\ de Computación. Facultad de Ciencias. Universidad del Zulia. Maracaibo, Venezuela. \\ Acreditado en el Programa de Promoción al Investigador Nivel II. \\ E-mail: jandrade@luz.ve, jandrade01@yahoo.com
}

\section{Resumen}

El propósito de esta investigación es realizar una contribución teórica al tema de las tecnologías y los sistemas de información (TSI) en las organizaciones, en relación con dos factores: técnicos y sociales. Se trata de una contribución basada en el análisis racional; este procedimiento metodológico permite comprender cómo se produce conocimiento en las organizaciones con ayuda de las TSI. Se analiza si en el desarrollo de conocimiento, las TSI como factores técnico de las organizaciones, incorporan las estructuras sociales (normas, procedimientos, reglas) o si estas estructuras son afectadas por el uso de las TSI. Se plantea que tecnología y usuarios se influyen mutuamente en un proceso continuo de gestión e innovación de conocimiento que institucionaliza las estructuras de la organización a través de procesos que legitiman el poder. En tal sentido, se cuestiona si la naturaleza del conocimiento es afectada por los aspectos sociales y políticos que subyacen en las organizaciones. Parte de las conclusiones que se alcanzan es la de entender que los sistemas y tecnologías de información son herramientas que legitiman el poder en las organizaciones.

Palabras clave: Tecnologías y sistemas de información, gestión del conocimiento, organizaciones, legitimación.

\section{I nformational Technologies and Systems in Knowledge Management in Organizations}

\begin{abstract}
The purpose of this research is to contribute to theory related to the theme of technology and information systems (TSI) in organizations.in relation to social and
\end{abstract}


technical factors. It is a constribution based on rational analysis, and the methodological procedure permitted the acquisition of knowledge as to how knowledge is produced in organizations through the use of TSI. The development of knowledge is studied in organizations to see if the TSI are technical factors in organizations, which incorporate social structures (norms, procedures, rules) or whether these structures are affected by the use of TSI. It is proposed that both technology and users are mutually affected in a process of continuous knowledge management and innovation that institutionalize the organizational structures through processes of power legitimization. In this sense, whether the nature of knowledge is affected by social and political aspects that underlie organizations is questioned. One of the conclusions reached is that information system technologies are tools that legitimize power in organizations.

Keywords: Information systems technologies, knowledge management, organizations, legitimizing power.

Recibido: 03-07-31. Aceptado: 03-10-17

\section{I ntroducción}

Variadas reflexiones dieron origen a esta investigación, y para comprender la tesis central del análisis, es menester precisar algunos conceptos fundamentales que permitan enfatizar en los aspectos teóricos que subyacen en el an álisis organizacional, en lugar de concentrarse en los elementos empiristas, tradicionalmente desarrollados cuando se analizan los aspectos asociados a las tecnologías y sistemas de información -TSI-.

Los investigadores - con frecuencia- se aproximan al estudio de las organizaciones para resolver problemas aplicados, sin darle mucha importancia a los aspectos teóricos que subyacen en el análisis; por ejemplo, cuando se estudian aspectos relacionados con las tecnologías de información, una gran mayoría de las investigaciones se concentra en el análisis del grado de aceptación de las tecnologías y sistemas de información, en el impacto que ellas tienen sobre los sistemas y sobre la gente o en las resistencias de los usuarios para utilizar los nuevos procesos y sistemas. Esas y otras formas de desarrollar investigación empírica descuidan o minimizan la importancia de analizar las teorías que subyacen en los distintos análisis.

En esta investigación trato el tema de los sistemas de información desde una perspectiva social. No asumo el enfoque técnico tradicional en el cual se suele medir el impacto que una variable como la tecnología tiene sobre la sociedad, lo que constituye, a mi juicio, una 
visión excesivamente unidireccional. Es una investigación teórica del tema de los sistemas de información en las organizaciones, con una visión humanista donde prevalecen por igual los elementos meramente técnicos con los factores sociales. En esta investigación pongo un fuerte énfasis en el desarrollo teórico; y para ello, analizo los sistemas de información en el contexto de las organizaciones.

Al estudiar las organizaciones, abordo los sistemas de información desde un nuevo enfoque organizacional. Afirmo que es nuevo porque se trata de un análisis de los sistemas de información desde un punto de vista conceptual, en oposición al tradicional análisis de aspectos técnicos que ha dominado, hasta ahora, el estudio de esta disciplina. Pretendo discutir, si las tecnologías de información son determinantes para la aplicación y desarrollo de nuevos procesos, y si ellas tienen utilidad en cuanto a la gestión de conocimiento que se utiliza. En ausencia de estudios que analicen las TSI más allá de su practicidad, yo estudio la dinámica de los sistemas de información a fin de entender cómo se usan y cómo se estructuran en la organización, y si los sistemas son desarrollados e incorporados a la organización en función de las nuevas tecnologías de información. También analizo si las nuevas tecnologías de información impactan las estructuras organizacionales o si por el contrario, ellas lo que hacen es incorporar o representar las estructuras sociales de la organización.

Por lo tanto, me propongo demostrar que es útil e importante tener un punto de vista más amplio del fenómeno de los sistemas de información, con una visión que pondere por igual lo subjetivo (social-político) y lo objetivo (técnico). El análisis que hago de los sistemas y tecnologías de información en las organizaciones me permite interpretarlos como acoplados con la actividad humana, de forma tal que ellos son el resultado de acciones humanas coordinadas y por lo tanto los interpreto como inherentemente sociales. Así, podemos observar el rol de los sistemas de información en términos de la existencia de una interacción mutua entre los agentes humanos y la tecnología.

Bajo una consideración ontológica acerca de la naturaleza del conocimiento de los sistemas de información, se requiere de una teoría que posibilite cuestionar si la naturaleza del cómo se conoce, con el uso de TSI, es afectada por los aspectos sociales y políticos que subyacen en las organizaciones. Por lo tanto, para entender el análisis se requiere de una herramienta que permita el desarrollo de sistemas de información que sea sensible a esos aspectos. Esa herramienta está basada en una teoría de corte sociológico que se denomina Teoría de la Estructuración desarrollada por Anthony Giddens.

Utilizando los elementos conceptuales de la Teoría de la Estructuración, argumento que 
el poder media para gestionar lo que se conoce y el cómo se conoce, cómo el conocimiento es institucionalizado en las organizaciones con el apoyo de TSI y cómo las relaciones estructurales utilizan el conocimiento como mecanismo para reafirmar el poder. Por lo tanto, esta investigación define los fundamentos teóricos que sostienen la importancia de estudiar las organizaciones cuando se incorporan TSI, a la vez que analizo cómo éstas afectan y son afectadas por las estructuras y estrategias que involucran el manejo o gestión del conocimiento organizacional.

\section{Sistemas y tecnologías de información con perspectiva social}

La investigación en sistemas de información y en organizaciones está fuertemente influenciada por los modelos estructurales y estratégicos, que no son siempre muy eficientes en la práctica. Son modelos analíticos funcionalistas, que intentan resolver problemas de funcionamiento gerencial atacando las dificultades que se dan en las estructuras de la organización. Estos modelos se utilizan porque primero, se asume que existe un problema que está muy bien definido y puede ser resuelto, y segundo, porque los factores políticos, sociales y culturales no generan consecuencias sobre el problema. Las organizaciones de hoy se estructuran alrededor de la información, y la usan no solamente como un recurso estratégico para obtener ventajas, sino como objeto mismo de configuración de dinamismos gerenciales. En ese contexto, el érmino Sistemas de Información en forma general denota sistemas de computación, o algún tipo de sistema mecánico o electrónico donde está presente la información. Más recientemente se le conoce como sistemas asociados a las tecnologías de información y comunicaciones. El dominio de los sistemas de información es aquel que está orientado a proveer información; en ambientes organizacionales, trata de la colección, almacenamiento y diseminación de información relevante para la toma de decisiones, por intermedio del uso de tecnologías digitales.

En sus aspectos prácticos, los sistemas de información se estudian observando los flujos de información existentes en las organizaciones, esto se hace con el fin de planificar y diseñar nuevas formas de mejorar la eficiencia para la toma de decisiones. Este tipo de enfoque pragmático considera que buenos sistemas de flujos de datos suponen una mejora en la calidad de la información que se requiere. Bajo esta perspectiva, los aspectos técnicos son los más relevantes, y por lo tanto, cualquier otra consideración que signifique el análisis profundo de las dimensiones sociales y políticas no tiene efecto directo en el ulterior objetivo de los sistemas de información.

Sin menoscabo de la necesidad de estudiar y desarrollar sistemas constituidos por 
factores técnicos, puedo afirmar que existe una notable ignorancia acerca de la aplicabilidad de los diversos conceptos sociales que relacionan TSI con organizaciones; lo cual es explicable porque el estudio de los sistemas de información es visto como perteneciente al campo del desarrollo de aplicaciones de sistemas en organizaciones. Las aplicaciones se caracterizan por la existencia de desacuerdos metodológicos que se utilizan en la amplia variedad de técnicas disponibles, y por las inexactitudes y maneras fragmentadas de desarrollar las aplicaciones. Tales desacuerdos metodológicos surgen porque el estudio de los sistemas de información se hace en función de los elementos prácticos, en lugar de los aspectos ontológicos y filosóficos que subyacen en los particulares enfoques de investigación. Se le da prioridad al uso práctico de los sistemas de información en detrimento de las posturas epistemológicas que permiten conocer la fuente de conocimiento. Por lo tanto, existe la necesidad de investigar enfoques alternativos del desarrollo de sistemas de información orientados a las organizaciones.

El estudio de los sistemas de información es concebido, por muchos autores, como un área multidisciplinaria que en particular, cuando refiere al campo de la información en el contexto social, trata de vincular las tecnologías con el área humanística de las organizaciones, especialmente en lo atinente a la gerencia de información y a la gestión de conocimiento organizacional.

Por consiguiente, no es extraño encontrar una tendencia creciente en la utilización de procesos, aplicaciones de software, sistemas y tecnologías de información, que va acompañado con el reordenamiento de las estructuras de las organizaciones. Ese reordenamiento de las estructuras organizacionales se posibilita a través de posiciones ideológicas que se manifiestan en el desarrollo de los sistemas y procedimientos.

Los sistemas de información, junto a las herramientas y tecnologías que se utilizan en el desarrollo de aplicaciones, llevan en su seno elementos ideológicos que se solidifican con el discurso gerencial. A nivel práctico, los gerentes no actúan solos, sino que son apoyados por una variedad de ideas, teorías, modelos y esquemas, que son generados para ellos por los "teóricos" gerenciales, bien sean especialistas, consultores o académicos. Así, las ideas del discurso ayudan a legitimar las existentes estructuras de poder y a acentuar las inequidades que se presentan en la distribución de los recursos de las organizaciones. En relación con los aspectos personales, se pudiera explorar cómo funciona lo ideológico a nivel consciente, observando las manipulaciones que se hacen a los sistemas y comunicaciones; pero en un plano institucional, se puede analizar el poder, a través de las ideas, discursos y conocimiento que se utiliza para favorecer los intereses dominantes. Es así como las ideas y discursos acentúan las relaciones de poder que se 
manifiestan en las estructuras de toda organización.

\section{Estructuración del poder en las organizaciones}

Debido a que es relevante la participación de la información sobre el eje alrededor del cual se reestructuran las estrategias, se generan y concretan nuevas formas de producción y de división del trabajo, es relevante también, el despliegue de mecanismos que generan un reordenación del espacio organizacional.

Detrás de cualquier práctica organizacional ha existido la necesidad de alcanzar una práctica intelectual que defina el estudio de las organizaciones, a través de un entendimiento que permita el proceso de constitución de la sociedad moderna (Reed, 1993, citado por Dávila). Aunque existe una fuerte tendencia a clasificar a las organizaciones en términos de sistemas técnicos, la organización moderna no es meramente un sistema técnico o social, sino que requiere de la estructuración e integración de las actividades humanas alrededor de la tecnología y sistemas de información. Es así como, la necesidad de entender el tema de la organización desde un punto de vista teórico, pasa por una práctica organizacional que es en esencia social.

Max Weber (1947, citado por Pugh y Hickson, 1989) definió a las organizaciones, en un sentido sociológico, como un sistema de actividades humanas dirigidas continuamente hacia una meta específica. La contribución principal de Weber al estudio de las organizaciones fue su teoría de la estructura de la autoridad, que lo condujo a caracterizar a la organización en términos de relaciones de autoridad. Weber hizo una distinción entre poder, la habilidad de forzar a la gente a obedecer (sin considerar sus resistencias), y autoridad, donde las órdenes son voluntariamente obedecidas por quienes las reciben. Weber distingue entre tipos de organizaciones de acuerdo a la forma en que la autoridad es legitimada. Considerado en forma abstracta, los hombres en la sociedad actúan en contra de los otros en base a sus intereses materiales y a sus ideales, estableciendo una relación de autoridad y obediencia en función de entendimientos compartidos. Desde entonces, el pensamiento de Weber ha estado presente, de alguna manera, tanto en la práctica diaria como en el análisis de los estudiosos de la organización. Otros teóricos (Kahn, 1964; Jackson y Carter, 1991) han sugerido que el concepto de organización podría no existir si no fuera por las relaciones de poder. Mintzberg (1999) expresa que en el diseño de las estructuras organizacionales entra en juego una serie de factores de poder, particularmente la de un control externo de la organización, las necesidades personales de los distintos miembros y la moda del momento arraigada en la cultura donde se halla la organización (el poder de las normas sociales). Para Foucault (1997) las 
organizaciones son lugares de violencia controlada, donde el poder involucra a todos quienes participan en ellas. Foucault pensaba que el estudio de las cárcel como organización, podría iluminar cómo otras funcionan. Foucault argumentaba que el conocimiento es producido a través del discurso y para entenderlo es necesario examinar las instituciones que lo producen, así como las prácticas que sirven para crear no sólo el conocimiento, sino también el poder. Poder y conocimiento son para Foucault dos lados de la misma relación social.

La característica más resaltante en esas distintas concepciones teóricas es la presencia de estructuras sociales como parte integrante de la organización. Las estructuras sociales son tan diversas que se superponen a las estructuras técnicas de las organizaciones. $Y$ junto a tales complejidades, la acelerada interconexión de personas, gracias al uso de tecnologías de información y comunicaciones, genera situaciones que impulsan cambios en las estructuras sociales y en las técnicas usadas en las organizaciones.

Proponer una noción de organización que esté constituida por una teoría que conduzca a una práctica intelectual y social, y que a la vez sirva para acoplar e integrar otras prácticas sociales, se expresa muy adecuadamente en la definición de Giddens (en Dávila, 1997) quien enfatiza que la noción de organización como práctica intelectual no se refiere tanto a la distinción que hace referencia a una organización en particular, sino la de observar por intermedio de las organizaciones (como proceso societal) el devenir propio de la modernidad.

“Lo que distingue a las organizaciones modernas no es tanto su tamaño o su carácter burocrático, como el monitoreo reflexivo y concentrado que dicho tamaño y carácter burocrático lleva consigo. Quien dice modernidad no dice justamente organizaciones sino organización; es decir, el control regularizado de las relaciones sociales a través de indefinidas “distancias" espacio-temporales" (Giddens, 1991:16, citado por Dávila, 1997). $Y$ es que las organizaciones son sistemas que desarrollan procesos de datos que involucran la construcción social de los asuntos organizacionales, a través del diseño, desarrollo y aplicación de sistemas de información.

La organización es el lugar donde los procesos productivos se organizan con apoyo de tecnologías y sistemas de información, y que generan una indefinición de los conceptos de espacio y tiempo. Tales procesos son rutinizados a través de la práctica diaria. Al rutinizar los procesos, las estructuras sociales se incorporan a ellos y se institucionalizan a través de reglas, por medio de un proceso que se denomina estructuración. Con la práctica cotidiana, tales reglas se incorporan a las tecnologías y sistemas en forma de 
representaciones, para que las propias organizaciones las utilicen.

Así, por medio de un proceso muy bien tipificado de rutinización, se alcanza la institucionalización de los procesos y sistemas que se utilizan en la organización. La institucionalización se logra a través de hábitos que nos permiten ampliar el espectro de opciones que tenemos. Es por ello que, las organizaciones tienen institucionalizadas las normas y procedimientos que, por lo general, se expresan a través de los hábitos.

En la Teoría de la Estructuración, Giddens (1989) propone que las propiedades institucionales y la acción social se influyen las unas a las otras en una forma recursiva. Por consiguiente, las propiedades institucionales no existen fuera de la acción social. Ellas existen sólo porque son usadas por los agentes humanos. Así, los sistemas de información son causa y consecuencia de estructuras sociales.

La organización se convierte en un proceso que involucra una interacción mutua entre actores humanos y las propiedades estructurales de la organización, donde la acción humana se capacita a través de las estructuras y éstas, a su vez, son el resultado de la acción humana. Los sistemas y tecnologías de información posibilitan la legitimación de las estructuras de la organización. De esta manera, las organizaciones son el sitio donde nosotros como seres humanos actuamos y, por medio de esas actuaciones, cambiamos las estructuras del ambiente donde nos desenvolvemos con el apoyo de sistemas, tecnologías y rutinas que legitiman la estructura de la organización. Cuando los sistemas de información se institucionalizan y las estructuras se legitiman se consolida el poder organizacional; de tal forma que la legitimación y la institucionalización son rasgos propios del ejercicio del poder en las organizaciones.

Para conocer si un sistema de información está institucionalizado se debe examinar su dimensión política a fin de descubrir las relaciones de poder existentes en la organización. Cuando se examina el fenómeno del poder y este se enlaza a los sistemas de información, se puede entender el proceso de institucionalización de los sistemas de información y la gestión de un particular tipo de conocimiento. En la concepción de poder de Foucault (1980) no se emplean las usuales categorías y conceptos de individuos, estructuras, intereses e ideologías, ni siquiera se llega a definir el poder como tal. El poder no es un concepto que puede ser aislado y medido. El poder existe solamente en términos relacionales o cuando es expresado en términos de acción, por lo tanto el poder se ejerce en término de las relaciones, sin importar quien lo ejerce. El poder es una máquina que todo el mundo es agarrado, tanto por quienes lo ejercen, como por todos aquellos sobre quienes es ejercido (Foucault, 1980). 
Las relaciones de poder pueden ser mejor entendidas a través de la formas y técnicas en las cuales ellas son expresadas o representadas, en particular, a través de las restricciones que la gente se impone a sí misma e impone a los demás. El poder es expresado en los distintos microcontextos donde nos movemos y no se limita sólo a una particular entidad o dimensión, o a los privilegios que se nos otorga por una determinada posición que asumamos. El poder es ejercido en lugar de ser poseído, y en ese contexto, los sistemas de información son mecanismos de legitimación de poder debido, principalmente, al ejercicio que se establece entre las relaciones que se expresan en las acciones cotidianas.

\section{Legitimación e institucionalización de los sistemas de información}

El desarrollo de sistemas de información es, con frecuencia, conceptualizado como dos procesos mutuamente dependientes, pero claramente separados. Ellos son: uno de orden social, cuando se analiza el contexto organizacional, y el otro, de orden técnico, cuando se diseñan e implementan los sistemas de información para apoyar la estructura organizacional existente.

Bajo una visión humanista, los sistemas de información deben ser entendidos como los recursos materiales (tecnologías) que apoyan la práctica informacional de las organizaciones, que a su vez reafirma la práctica social en general. Cuando nos concentramos en los aspectos sociales y humanísticos de los sistemas de información, se adopta una postura interpretativa que considera los valores, las creencias y la construcción social de significados.

Como producto de la acción humana, los sistemas de información reflejan las estructuras del sistema social que intentan servir. Las organizaciones, junto a los individuos que la pueblan, forman parte de una red de valores, normas y reglas, donde el discurso es utilizado para legitimar sus estructuras socio técnicas. Por consiguiente, cada sistema es entendido como el conjunto de prácticas sociales que al estar rutinizadas, se institucionalizan a través de la cotidianidad organizacional. Los elementos institucionalizados de las organizaciones representan restricciones a las opciones que los individuos y las colectividades tienen.

Las tecnologías y sistemas de información, en general, pueden ser vistos como una restricción del contexto la cual produce alguna forma de reacción organizacional (Ranson, Hinings y Greenwood, 1980, citado por Orlikowski, y Robey, 1991), que a la vez de 
rutinizar procesos, permite a los seres humanos, a través de la práctica cotidiana, desarrollar hábitos que se institucionalizan en la organización.

Una importante propensión de las modernas organizaciones de hoy, en relación al desarrollo de sistemas y tecnologías de información, es la de "empacar" las tecnologías de información y sus aplicaciones junto a un discurso legitimador que en forma directa o velada institucionaliza procesos, aplicaciones y da pie a la utilización de tecnologías específicas en los ambientes organizacionales. El discurso es, por lo tanto, el método que observa, moldea y controla la conducta de los miembros de una organización. Es por ello que el mercado de las tecnologías de información es muy propenso a estandarizar los productos, a fin de ofrecerlos a una gran variedad de organizaciones, independientemente de su tipología o estructura. La oferta de tecnologías y sistemas -por lo general- va acompañada por una doctrina discursiva que la legitima.

Desde la década de los sesenta, las tecnologías de información ocuparon un lugar predominante en el espectro organizacional, delineando el horizonte del diseño de las organizaciones, caracterizado por una permanente innovación en las tecnologías de información y en la forma como las organizaciones realizan las tareas para alcanzar sus metas y objetivos (Andrade y Campo-Redondo, 1998). Tales diseños y tecnologías son acompañados con una doctrina y discurso que los legitima. Así por ejemplo, muchas aplicaciones de tecnologías de información a principio y mediados de la década de los noventa, fueron estimulados o justificados por una doctrina y discurso que se conoció como "reingeniería de procesos", el cual fue altamente influenciado por los trabajos de Hammer y Champy (1994). Desde sus comienzos, la reingeniería de procesos se planteo que todas las viejas estructuras deberían ser "barridas" radicalmente a favor del diseño de nuevos procesos organizacionales. Para alcanzar tal fin, se desarrollaron metodologías y herramientas de software para analizar procesos y seguimiento de eventos en forma visual y gráfica, desde variadas perspectivas: cliente, consumidor, producto, usuarios en la implantación de nuevos procesos, etcétera. Particular atención se prestó al desarrollo de software de simulación de modelos computacionales de los sistemas existentes y propuestos en las organizaciones; ello con el fin de probar la conducta de tales sistemas bajo condiciones específicas. El uso de esas herramientas sirvió para legitimar el discurso y la doctrina propia de la reingeniería de procesos, y para que la gerencia alta y media dominará mucho más la toma de decisiones en las organizaciones. De esa forma la noción de reingeniería funcionó como un proceso ideológico donde se conciliaron las contradicciones, conflictos e intereses que se perpetuaron en las organizaciones basados en la dominación y el poder. 
Pero no sólo, la reingeniería de procesos ha legitimado el uso de ciertas tecnologías de información; también lo han hecho las técnicas organizacionales de control de calidad, outsourcing, procesos de planificación estratégica, mejoramiento continuo, y más recientemente la gerencia o gestión de conocimiento (GC) que también intenta legitimar su discurso a través del uso de tecnologías de información. El acoplamiento entre tecnologías de información y los conceptos e ideas de cada uno de los enfoques gerenciales mencionados, por lo general, se centra en el comportamiento que las organizaciones desarrollan en función del incremento de la productividad. Las tecnologías de información se incorporan a las organizaciones asociadas a un particular discurso técnico que es permeado en todos los ambientes donde ella pueda legitimar su existencia, y así extender las formas en que pueden ser aplicados, para participar en el diseño y desarrollo de la organización y de sus estrategias.

La Figura 1 muestra cómo la práctica social recurrente, al utilizar las tecnologías de información, reproduce los procedimientos, estructuras sociales, reglas, tareas, normas y valores que los agentes a través de la rutinización en forma constante se apropian en forma de conocimiento, lo que les permite asumir en forma consciente sus acciones. Tal recurrencia se expresa en los diversos ambientes de la organización (político, social, económico, cultural) a través del proceso de institucionalización. La gestión de conocimiento (GC) se centra en prácticas organizacionales que se asocian al desarrollo de las TSI. El creciente énfasis puesto por las organizaciones en el desarrollo de la gestión de conocimiento supone que la fuente fundamental de productividad en la sociedad consiste en dominar el proceso de conocer. En tal sentido, la GC se centra en los problemas asociados a un diseño organizacional que le da prioridad al saber cómo hacer las cosas, en lugar de centrarse en los anteriores factores de producción como lo son el capital y el trabajo.

La gestión de conocimiento se relaciona con el esfuerzo por extraer y afianzar el conocimiento individual para ponerlo a disposición de los recursos de la organización. En ese proceso de extraer el conocimiento individual, la rutinización, tanto en el uso de los sistemas de información, como en el desarrollo de prácticas sociales, permite que se institucionalicen las estructuras, reglas, valores y normas de la organización. 
Figura 1

Institucionalización de la gestión de Conocimiento -GC-

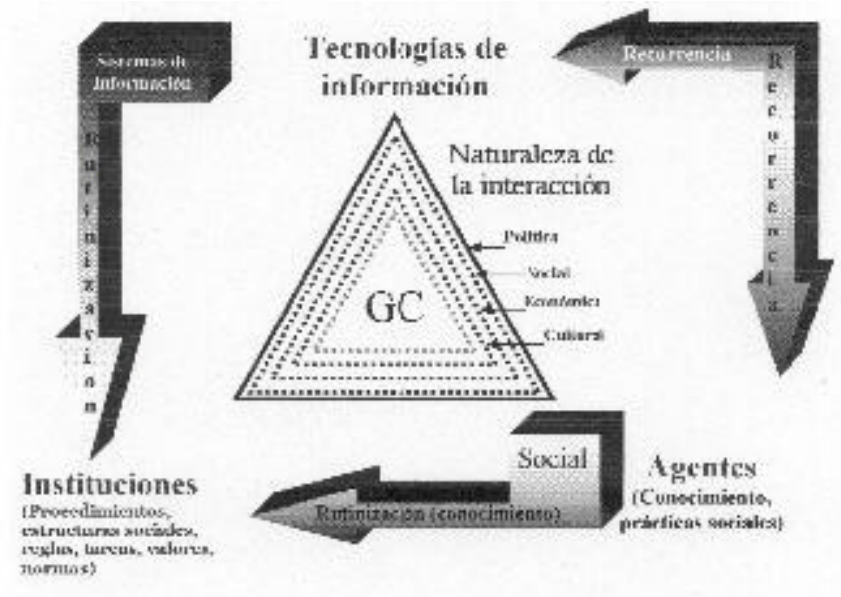

Fuente: Elaboración propia.

Empacar tecnologías de información y discursos requiere un incremento constante de procesos de innovación tecnológica, que cuando se adoptan y se aplican en una determinada organización conlleva al desarrollo de discursos gerenciales que justifica la transferencia y uso en otras organizaciones y por lo tanto abarca un espectro mayor, lo cual legitima aún más su existencia. Tecnologías y sistemas de información, en concordancia con el discurso técnico, se convierten en un factor de productividad que por medio de un proceso ideológico institucionaliza las estructuras del poder organizacional.

\section{Estructuración de las tecnologías y sistemas de información}

Los rasgos institucionalizados de los sistemas tienen propiedades estructurales debido a que las relaciones que se producen se estabilizan a través del tiempo y el espacio. Sin embargo, el supuesto que las relaciones se estabilizan debe ser objeto de duda cuando se trata de tecnologías de información, puesto que las TSI son esencialmente dinámicas y transformadoras. Al menos ello ocurre en la práctica diaria tal como lo percibimos en la velocidad del cambio tecnológico.

Algunos autores (Poole y DeSanctis, 1990, 1992; DeSanctis y Poole, 1994; Orlikowski y Robey, 1991) han afirmado que las tecnologías adquieren estructuras sociales que luego son apropiadas por lo usuarios durante su uso. Según estos autores, la gente, a través de la acción social, selecciona cómo la tecnología es diseñada y usada (DeSanctis y Poole, 1994). Esta manera de estudiar los fenómenos asociados a las tecnologías, enmarca la acción humana en términos de interacciones que ocurren entre tecnología y humanos. 
Según este punto de vista, las tecnologías tienen “incrustadas" las estructuras sociales las cuales han sido transferidas a través de su uso. Esa orientación concibe al uso de la tecnología como apropiadora de estructuras sociales; de tal forma que, la acción humana posibilita que las estructuras de la organización se manifiesten en las tecnologías y sistemas de información.

Por lo tanto, bajo la perspectiva de la estructuración, las tecnologías y sistemas son vistos como elementos estructurales de las organizaciones sociales. En base a lo anterior, se presupone que las tecnologías tienen embebidas o incrustadas ciertas estructuras (incluyendo las sociales) que existen previo a su uso. Sin embargo, esa posición es dudosa puesto que, la tecnología lo que hace es incorporar las reglas que gobiernan a la organización o más ampliamente a la sociedad y no a las estructuras que la conforman. Las estructuras están presentes en la tecnología en una forma virtual; por lo tanto, las reglas lo que hacen es manifestarse en forma de propiedades tecnológicas. La tecnología por si misma no encarna las estructuras de la sociedad, sólo las representan a través de sus propiedades. Por ejemplo, cuando un programador desarrolla un algoritmo o programa de computación, ese algoritmo se construye basado en un conjunto de reglas que involucra un lenguaje que tiene su propia estructura. Sin embargo, cuando esa persona utiliza un lenguaje de programación en particular probablemente no se da cuenta que está "representando" unas reglas que sirven para darle sentido al algoritmo que intenta desarrollar.

Las estructuras de las organizaciones (sociales) en lugar de ser estructuras fijas incorporadas a la tecnología, aparecen representadas en la práctica diaria. Por lo tanto, en lugar de concebir a la tecnología como apropiadora de estructuras, el enfoque que utilizo ve que las TSI "representan" las estructuras sociales a través de la incorporación de propiedades. De tal forma que, las tecnologías incorporan las representaciones de las estructuras sociales (ejemplo: datos almacenados, procedimientos, programas, rutinas, normas, etc.) que se convierten en propiedades de las reglas que se usan. Una vez que esas reglas se incorporan como propiedades a los sistemas, pasan a formar parte constitutiva de esos sistemas. Con el uso rutinario, esas propiedades dejan de ser reglas y por lo tanto no se pueden percibir como estructuras. De esta manera, la tecnología aparece en dos formas: como artefactos reconocidos aceptados socialmente (hardware, software, técnicas, procesos, etc.) y a través del uso que la gente hace de esos artefactos en sus prácticas diarias. Por ello, las estructuras están representadas en los sistemas y tecnologías y se manifiestan en la medida en que ellas son usadas socialmente.

Los elementos incorporados a las tecnologías que no se practican en la cotidianidad de 
las acciones humanas, no pueden ser considerados como parte de las estructuras de la organización o de la sociedad. Las estructuras que se plasman con el uso de tecnología son constituidas recursivamente en la regularidad de las acciones humanas, y es de esta forma como se moldea el conjunto de reglas que rigen las interacciones. Mientras más se utiliza las TSI a su plena capacidad, más se representan las reglas con que la tecnología fue diseñada. Su uso es considerado como una apropiación de las estructuras que están incorporadas en las tecnologías, y que la gente decide cómo usar.

En la Figura 2 señala cómo los actores (en forma grupal, individual u organizacional) se interrelacionan entre ellos a través de los recursos disponibles, y cómo las propiedades institucionales (incluyendo las dimensiones estructurales y culturales) se reafirman a través de los mecanismos de control, procedimientos estandarizados, normas, reglas y patrones de comunicación. Los componentes pueden ser percibidos por los actores (conscientes o inconscientemente) de distintas formas, pero esa percepción está mediada por el poder que se adquiere a través del conocimiento que se transfiere en la recursividad de las acciones cotidianas.

A través de la acción humana es que las estructuras se manifiestan en la tecnología. Cuando los seres humanos interactúan con tecnología, se comprometen con ciertas propiedades de ellas, generando una estructuración a través de las repetidas interacciones que se realizan (rutinización). Tal repetición expresada en una práctica social recurrente produce y reproduce partes de las estructuras de la tecnología. Cuando los elementos son rutinizados en la práctica diaria, entonces pueden ser parte estructural de la acción humana, y por lo tanto están disponibles para su transferencia. El resultado de una práctica social recurrente produce y reproduce una particular estructura que la tecnología usa (Orlikowski, 2000).

\section{Gerencia del conocimiento y los sistemas de información}

Las tecnologías y sistemas de información -junto a las telecomunicaciones- son herramientas importantes para la comunicación y el intercambio de información, tanto para las personas, como para las sociedades que trabajan para su propio desarrollo. Organizaciones de todo tipo: comerciales, no lucrativas, gubernamentales, públicas, privadas se basan en flujos de información y conocimiento. Las organizaciones de los sectores que participan en el desarrollo económico y social reconocen que el conocimiento es un pilar para alcanzar igualdad social para las regiones menos desarrolladas. $Y$ ello es debido a que se ha sembrado la esperanza de que con las nuevas tecnologías de información se puede alcanzar el beneficio social, puesto que se asume que la falta o 
deficiencia de conocimiento es responsable del retraso por alcanzar el desarrollo, no sólo de las organizaciones en forma individual sino de la sociedad en general.

Bajo esa perspectiva, las tecnologías y sistemas de información junto a las telecomunicaciones son vistas como herramientas neutrales que posibilitan la gestión y transferencia de conocimiento. Esa concepción neutral que se le otorga a las tecnologías y sistemas de información forma parte de un discurso modernista que niega la existencia de un comportamiento político intrínseco a la propia tecnología. No obstante, los procesos del diseño y uso de tecnologías de información no pueden ser abstraídos de las estructuras mismas de las organizaciones, o de los roles desempeñados por sus diseñadores y usuarios dentro de esa particular estructura organizacional.

Figura 2

\section{Gerencia de los sistemas de información y los procesos de transferencia de conocimiento}

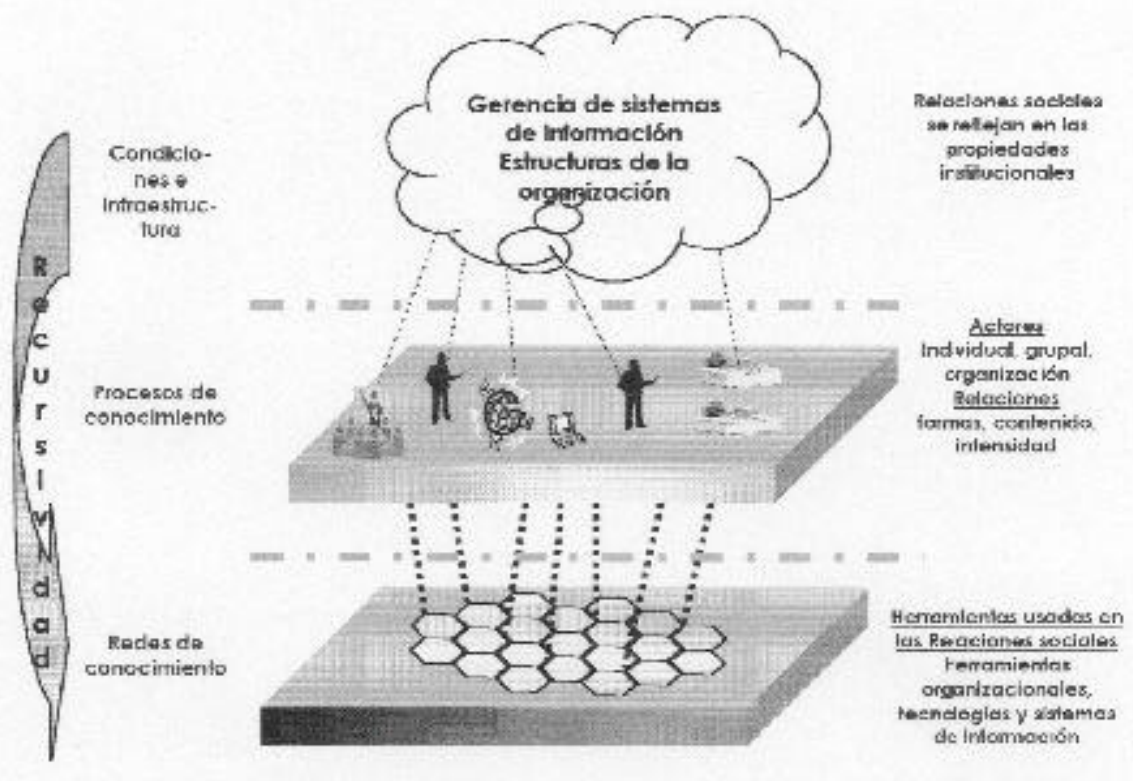

Fuente: Elaboración propia.

Las organizaciones no solamente son moldeadas por las tecnologías de información, sino que son altamente influenciadas por procesos políticos y sociales y por las acciones de los miembros de las organizaciones. Ello es así, porque detrás de la tecnología existe una política y toda política tecnológica afecta el desarrollo difusión y uso de la tecnología. Las nuevas TSI tienen profundo impacto en la experiencia organizacional, por ello uno de las funciones de la organización es la de minimizar los impactos negativos que el cambio tecnológico puede ocasionar. 
Durante los últimos diez años, la adopción de novedosos sistemas y tecnologías de información en organizaciones ha llevado implícita una poderosa carga cultural, puesto que ha envuelto a un amplio espectro de actores y situaciones que son insertados en la vida organizacional en forma material y discursiva. Las herramientas para la gerencia de conocimiento, desarrolladas a finales de la cécada de los noventa, aparecieron para la solución de problemas que se presentaban en organizaciones del sector privado, particularmente de los Estados Unidos; pero su discurso se transfirió a organizaciones gubernamentales y otras de alcance internacional no privadas, y que, en la actualidad se expresan en términos y frases como "sociedad de la información", "sociedad del conocimiento", "nueva economía", "bancos de conocimiento" “info economía” y otros más. Esa incrementada tendencia de prestar atención a este tipo de modalidad gerencial refleja un movimiento organizacional desde el anterior discurso de reingeniería hacia un nuevo discurso asociado al conocimiento. El discurso de la nueva gerencia organizacional considera importante, aquellas actividades y habilidades para crear y gerenciar los activos del conocimiento en las organizaciones y la sociedad en general. Lo interesante de este cambio de discurso gerencial es que ha afectado a instituciones de carácter internacional que tienen pertinencia social o que están involucradas con nuevos modelos de desarrollo económicos y sociales, que en lo atinente al uso de los sistemas y tecnologías de información, se concentran en el análisis de la brecha que se produce entre los países con mayor capacidad y disponibilidad para alcanzar la participación plena de la población, y la de aquellos que no pueden participar en el nuevo concepto social de información y conocimiento.

Una clave vital para crear una organización basada en el conocimiento es el uso apropiado de tecnologías que le den soporte a las organizaciones (Kogut y Zander, 1992). La continua transformación socio-económica de la sociedad se basa en procesos interrelacionados de informatización y comunicaciones acoplados a estructuras organizacionales interdependientes y cambiantes con intenso uso de conocimiento. Iniciativas técnicas y organizacionales pueden proveer una infraestructura adecuada para apoyar los procesos de gerencia de conocimiento. Por lo tanto, es importante conocer cómo podemos usar las tecnologías de información de manera que sean innovadoras, de forma tal que permitan hacer las cosas en función del beneficio social.

La tecnología existe en un contexto de significados sociales prácticos y los cambios tecnológicos no pueden ser concebidos sin entender los procesos de cambio social. "EI proceso de creación de conocimiento es un proceso social que está inmerso en un conjunto particular de relaciones entre los individuos, equipos y organizaciones" (Nonaka 
y Nishiguchi, 2001:286). La información es un medio necesario para extraer y construir conocimiento (Nonaka y Takeuchi,1999). Al analizar los sistemas de información y el conocimiento es preciso concentrarse en los aspectos teóricos que conducen al entendimiento de los aspectos prácticos que existen en el contexto social, y entender también aquellas instituciones que son creadas a través de la práctica.

Desafortunadamente, muchas veces la gerencia de conocimiento se ha limitado a codificar y almacenar datos en estructuras tecnológicas cada vez más sofisticadas. Los datos almacenados representan observaciones o hechos fuera de contexto y por ende, sin significado intrínseco. Cuando los datos son colocados dentro de un contexto con significado entonces es posible tener información, frecuentemente en forma de mensajes. Se requiere que las organizaciones dejen de ser gestores de datos e información y promuevan la gestión de conocimiento, no sólo extraído de los individuos, sino de la organización como un todo. En tal sentido, la gerencia del conocimiento debe ser un mecanismo que permita a las organizaciones producir conocimiento en forma sistemática para fomentar la participación e innovación de ventajas estratégicas.

La necesidad de pertenecer a una sociedad de conocimiento ha incentivado a las organizaciones a desarrollar sistemas de información que intentan mejorar los procesos de transferencia de conocimiento, entendidos como aquellos "sistemas de información diseñados específicamente para facilitar la codificación, colección, integración y diseminación de conocimiento organizacional" (Alavi y Leidner, 1999:4) e inducir a la creación de formas de participación social que generen conocimientos en las organizaciones y que sean indispensables para alcanzar eficiencia, eficacia y competencia.

El discurso de la gerencia del conocimiento reconoce que la habilidad para gestionar los "activos del conocimiento" es importante para las organizaciones. Y para desarrollar tales habilidades se presta atención a los tecnologías y sistemas de información. Es así como se puede entender que ciertas tecnologías de información sean tan aceptadas y desarrolladas en términos de necesidades organizacionales. Nunca antes, la utilización de las tecnologías de información estuvo tan fuertemente influenciada por la dinámica social como ha ocurrido con el advenimiento de las redes de datos a través de lo que se conoce como internet. En las organizaciones, la tecnología que sustenta a la internet (TCP/IP) se ha implantado en dominios de información privada, lo cual ha permitido el desarrollo de bancos de datos o de conocimiento para manejo particular, de forma tal que su uso ha modificado las estructuras de la organización.

A través del uso intensivo de las tecnologías de información, se modifican las estructuras 
de la organización; por ello, la gerencia del conocimiento requiere un apoyo social para poderse desarrollar de manera que la tecnología no se convierta en una barrera para la gestión, diseminación y utilización del conocimiento.

\section{Reflexiones finales}

Este trabajo ha examinado importantes rasgos socio técnicos de los sistemas de información en las organizaciones, incluyendo el rol del discurso en la conformación de estructuras de poder institucional. Es indudable que el discurso ayuda a legitimar las estructuras de poder y a acentuar las inequidades que se presentan en la distribución de los recursos de las organizaciones. En el análisis, se establecieron tres importantes argumentos que indican que existe una relación dinámica entre las estructuras organizacionales y las tecnologías y sistemas de información -TSI-. El primer argumento señala que existe la necesidad de estudiar las tecnologías y sistemas de información con una visión humanista que incluya los aspectos ideológicos que intervienen en la recomposición de las estructuras de la organización. El segundo argumento refiere a la interpretación que se hace del uso de las tecnologías y sistemas de información, como herramientas que institucionalizan las estructuras de poder en la organización, a través de hábitos que nos permiten ampliar el espectro de opciones que se nos presentan. A través de la práctica social recurrente y por medio de un proceso de rutinización, las tecnologías de información ayudan a modificar y reproducir los procedimientos, estructuras sociales, reglas, tareas, normas y valores que prevalecen en la organización. El tercer argumento es que por medio de las tecnologías y sistemas de información se crea conocimiento que modifica e institucionaliza las estructuras de la organización. De manera que las tecnologías y sistemas de información incorporan las estructuras de poder de las organizaciones y se manifiestan a través del conocimiento que se crea. En ese proceso, las tecnologías existen en un contexto de significados sociales prácticos que no pueden ser concebidos sin entender los procesos de cambio social.

\section{Referencias Bibliográficas}

1. Alavi, Maryam y Leidner, Dorothy (1999), “Knowledge Management Systems: Issues, Challenges, and Benefits". Communications of the Association for Information Systems -AIS-, 1 (Febrero).

2. Andrade, Jesús Alberto y Campo-Redondo, María (1998), “Tecnologías de Información en el Diseño de la Organización". En: Revista Venezolana de Gerencia, Año 3, No 6, Venezuela. La Universidad del Zulia. Vice-rectorado Académico, pp 241-256. Maracaibo, 
Venezuela.

3. Dávila, Jorge (1997), “Teorías de las Organizaciones: Panorama Actual". Revista Venezolana de Gerencia. Año 2, No. 4. Diciembre, 1997. La Universidad del Zulia. Vicerrectorado Académico. Maracaibo, Venezuela.

4. DeSanctis, Gerardine y Poole, Marshall (1994), "Capturing the complexity in advanced technology use: Adaptive structuration theory". Organization Science. 5(2) 121-147.

5. Foucault, Michel (1980), Power/ knowledge. New York. Pantheon.

6. Foucault, Michel (1997), La arqueología del saber. Editorial Siglo XXI

7. Giddens, Anthony (1989), The constitution of society: Outline of a Theory of Structuration. Berkerly CA. University of California Press.

8. Hammer, Michael y Champy, James (1994), Reingeniería. Olvide lo que usted sabe de cómo hacer funcionar una empresa. ¡Casi todo está errado! Editorial Norma. Colombia.

9. Jackson, Norman y Carter, Pippa (1991), “In defence of paradigm incommensurability". Organisation Studies, 12 (1), 109-127.

10. Kahn, Robert (1964), Power and conflict in organizations. Ediciones R. L. Kahn \& E. Boulding. London: Tavistock. pp. 1-10.

11. Kogut, Bruce y Zander, Udo (1992), "Knowledge of the firm, combinative capabilities and the replication of technology". Organization Science, 3, 383-397.

12. Mintzberg, Henry (1999). La estructuración de las organizaciones. Ariel Economía. Barcelona, España.

13. Nonaka, I kujiro y Nishiguchi, Toshihiro (2001), “Conclusion. Social, technical, and evolutionary dimensions of knowledge creation". En Nonaka, I y Nishiguchi,T (Eds.). Knowledge Emergence. Social, technical, and evolutionary dimensions of knowledge creation ( $p p$ 286-289). Oxford Univesrity Press.

14. Nonaka, Ikujiro y Takeuchi, Hirotaka (1999), La organización creadora de 
conocimiento. Oxford University Press. Oxford.

15. Orlikowski, Wanda (2000), “Using Technology and Constituting Structures: A practice Lens for Studying Technology in Organizations". Organization Science. Vol. 11. No. 4 Julio-Agosto 2000, pp. 404-428.

16. Orlikowski, Wanda y Robey, Daniel (1991), “Information Technology and the Structuring of Organizations". Information Systems Research. Vol 2: 2. pp. 143-169. The Institute of Management Sciences.

17. Poole, Marshall y DeSanctis, Gerardine (1990), "Understanding the Use of Group Decision Support Systems: The Theory of Adaptive Structuration". In Fulk, J . \& Steinfield, C.W. (Eds.), Organizations and Communication Technology, Newbury Park CA: Sage 1990: 173-193.

18. Poole, Marshall y DeSanctis, Gerardine (1992), “Microlevel Structuration in Computer-Supported Group Decision Making", Human Communication Research, 19, 1, September 1992: 5-49.

19. Pugh, D y Hickson, D (1989), Writers on Organizations. Penguin Books. 